\title{
A Case of Relapsing-Remitting Neuroborreliosis? Challenges in the Differential Diagnosis of Recurrent Myelitis
}

\author{
P. Albrecht ${ }^{a} \quad$ N. Henke ${ }^{a} \quad$ H.C. Lehmann ${ }^{a} \quad$ S. Macht ${ }^{b}$ \\ H. Hefter ${ }^{a} \quad$ N. Goebels ${ }^{a} \quad$ C. Mackenzie ${ }^{c} \quad$ T.A. Rupprecht ${ }^{d}$ \\ V. Fingerle ${ }^{e}$ H.P. Hartung ${ }^{a} \quad$ A. Methner ${ }^{a}$ \\ Departments of ${ }^{\mathrm{a}}$ Neurology, ${ }^{\mathrm{b}}$ Diagnostics and Interventional Radiology, and \\ ${ }^{c}$ Medical Microbiology and Hospital Hygiene, Medical Faculty, Heinrich Heine \\ University, Düsseldorf, 'Department of Neurology, Amper Clinic Dachau, \\ Dachau, and ${ }^{\mathrm{e}}$ National Reference Center for Borrelia and Ehrlichia, Bayerisches \\ Landesamt für Gesundheit und Lebensmittelsicherheit, Oberschleissheim, \\ Germany
}

\section{Key Words}

Neuroborreliosis - Borrelia burgdorferi - Lyme borreliosis - Cerebrospinal fluid . Myelitis $\cdot$ CXCL13

\begin{abstract}
We report the case of a 31-year-old woman with 4 episodes of myelitis with pleocytosis, a positive Borrelia burgdorferi serology with positive antibody indices, and full recovery each time after antibiotic and steroid treatment, suggesting neuroborreliosis. We nevertheless believe that recurrent neuroborreliosis is improbable based on the levels of the chemokine CXCL13 in cerebrospinal fluid and favor the diagnosis of post-infectious autoimmunemediated transverse myelitis possibly triggered by an initial neuroborreliosis as the cause of the relapses observed in our patient. We demonstrate the diagnostic steps and procedures which were important in the differential diagnosis of this unusual and challenging case.
\end{abstract}

\section{Introduction}

Lyme borreliosis is a tick-borne anthropozoonosis caused by the spirochete Borrelia burgdorferi. Asymptomatic infections are frequent in Europe, as Borrelia-specific antibodies have been observed in $5-25 \%$ of healthy subjects [1-4]. The primary stage is characterized by erythema migrans and can be followed by dissemination of disease, 
which mainly affects the joints, heart or nervous system [4-6], within weeks to months. The most frequent neurological manifestation of Lyme borreliosis is meningopolyradiculoneuritis. Neuroborreliosis of the central nervous system (CNS) is rare, and differentiation from other disorders of the CNS with concomitant seropositivity for $B$. burgdorferi can be difficult.

Herein, we report a case with positive B. burgdorferi serology and 4 episodes of severe CNS manifestations with full recovery each time after sufficient antibiotic treatment. We illustrate the diagnostic steps and procedures which were valuable for posing the differential diagnosis in this unusual case, taking into account current stateof-the-art procedures for the diagnosis of Lyme borreliosis.

\section{Case History}

A 31-year-old woman presented with a 2-day history of rapidly progressing hypoesthesia of the body below the neck, tetraparesis, gait ataxia, brainstem symptoms, and urinary retention.

This was already the fourth episode of symmetric sensomotoric deficits of the limbs. Fifteen years earlier, in 1996, she had suffered from a mild paraparesis with hypoesthesia of both legs, which remitted completely after treatment with an oral antibiotic and glucocorticoids. Details were not remembered and neither cerebrospinal fluid (CSF) analysis nor MRI scans had been performed.

The second episode occurred 3 years later, in 1999, with acute urinary retention and a mild-tomoderate sensomotoric deficit below dermatome T6. Transcranial magnetic motor evoked potentials (MEP) and somatosensory evoked potentials (SSEP) were altered. Cerebral and spinal MRI scans were normal, but CSF analysis showed a lymphocytic pleocytosis of 260 leukocytes/ $\mu \mathrm{l}$, and a highly elevated B. burgdorferi CSF/serum IgG antibody index (AI) of 8.0 (normal <2.0), leading to the diagnosis of neuroborreliosis with transverse myelitis. The patient was subsequently treated intravenously for 14 days with ceftriaxone $2 \mathrm{~g}$ /day and prednisolone $80 \mathrm{mg} /$ day and recovered completely. Control CSF analysis showed a regression of the pleocytosis to 30 leukocytes/ $\mu \mathrm{l}$ and a reestablishment of the blood-brain barrier (BBB) function with an albumin CSF/serum quotient of 3.6 compared to 12.8 before treatment.

During the third episode in 2008, the patient developed neck pain, a mild gait ataxia, and paresthesia of both legs. Cerebral and spinal MRI scans, MEP and SSEP were normal, but CSF analysis revealed 293 leukocytes/ $\mu \mathrm{l}$ with a positive $B$. burgdorferi IgM (EIA and Western blot) in serum but not in CSF. The AI was reported to be normal. Treatment consisted of intravenous ceftriaxone $2 \mathrm{~g} / \mathrm{day}$, which was later switched to oral doxycycline $100 \mathrm{mg}$ bid and notably no corticosteroids. The patient made a complete clinical recovery, and CSF reanalysis after treatment showed a reduction of the pleocytosis to 15 leukocytes/ $\mu$ l.

The only other remarkable prior condition was an X-type histiocytosis with a strictly dermal manifestation diagnosed in 1982, which completely remitted and had not caused any further manifestation since treatment with methotrexate and Purinethol. Notably, the patient did not recall a tick bite or erythema migrans at any time.

\section{Neurological Symptoms and Diagnostic Findings on Admission}

On admission in March 2011, the patient presented with a sustained gaze-evoked nystagmus to the left and an incomplete abducens nerve paralysis. Motor examination revealed a moderate-to-severe symmetric tetraparesis. Reflexes were brisk in all extremities and the plantar response was neutral. The patient was not able to stand or walk, but showed moderate ataxia of all limbs. Sensory testing revealed sensory deficits below dermatome $\mathrm{C} 4$, with impaired discrimination of sharp and dull, and essentially no sense of vibration, temperature and position. She complained of urinary retention.

A spinal MRI scan revealed extensive signal alterations especially in the cervical, but also in the thoracic spinal cord. Some of these lesions comprised 2 or more segments and involved large parts of the cord's cross section (fig. 1a, b), in combination with moderate swelling at the cervical level and a 
faint enhancement after intravenous gadolinium administration (not shown). A cerebral MRI scan showed bilateral pontine $\mathrm{T}_{2}$ signal hyperintensities (fig. 1c).

CSF analysis revealed a predominantly lymphocytic pleocytosis of 136 leukocytes/ $\mu \mathrm{l}$; dysfunction of the blood-CSF barrier, with an elevated CSF/serum albumin quotient of 18.8; and an elevated total CSF protein of $126 \mathrm{mg} / \mathrm{dl}$. There were no oligoclonal bands, but a Reiber diagram suggested intrathecal synthesis of IgM (IgM quotient 11.96, IgM index 0.64) but not IgG (IgG quotient 12.78, IgG index 0.68). Measles, rubella, and zoster AIs (MRZ reaction <1.3) were negative. CSF/serum AIs of 52.6 and 18.8 were highly positive for B. burgdorferi IgM and IgG, respectively, with a B. burgdorferispecific IgM concentration of $18.9 \mathrm{U} / \mathrm{ml}$ and an IgG concentration of $1.3 \mathrm{U} / \mathrm{ml}$ in CSF. Western blotting was positive in CSF and serum for p41- and OspC-specific IgM and IgG at day 1, and revealed an additional positive 0sp17-IgM band in serum at days 7 and 17 (fig. 1d). Quantitative real-time PCRs amplifying the OspA gene and the P41 gene of B. burgdorferi sensu lato were negative in CSF. The workup was extended and included other differential diagnoses. Serology for Mycoplasma pneumoniae was positive in serum (immunohistochemical assay, 1:320; enzyme immunoassay, borderline positive) but not in CSF. Two different PCRs for detection of mycoplasma DNA were performed on CSF. A specific PCR for M. pneumoniae was negative. A PCR for 16S ribosomal DNA was positive, and the amplicon was cloned and sequenced but revealed no mycoplasma-specific sequences. Primer sequences can be obtained from the authors.

Four months after the episode, serological controls revealed a non-significant rise in $M$. pneumoniae titer in serum (immunohistochemical assay 1:640), with persistent negative results in CSF. Aquaporin-4 antibodies were negative, as were antinuclear antibodies, antineutrophil cytoplasmic antibodies, anti-DNA antibodies, anti-extractable nuclear antigens, HIV-, Treponema-, hepatitis B and C serology, and PCR of HSV, CMV, VZV and EBV in the CSF. Angiotensin converting enzyme activity in serum was normal.

The evoked potentials gave evidence of an affection of the central pyramidal tract and a demyelinating affection of the somatosensory afferents in both legs, while visual and acoustic evoked potentials were normal. Electroneurography excluded an involvement of the peripheral nervous system.

\section{Treatment and Course of Recovery}

The patient was treated with intravenous ceftriaxone $2 \mathrm{~g} /$ day, acyclovir $3 \times 750 \mathrm{mg} /$ day and methylprednisolone $1 \mathrm{~g} /$ day. Acyclovir was discontinued after a negative PCR for HSV and VZV DNA. Ceftriaxone was continued for 21 days. After 1 week, methylprednisolone was continued orally and tapered out over 2 weeks. The patient's clinical status ameliorated rapidly; she started to walk again after 1 week and was out of bed most of the day after 3 weeks.

CSF analysis after 7 days of treatment revealed a reduction of leukocytes to $26 / \mu \mathrm{l}$ along with a reduction of the total protein to $33 \mathrm{mg} / \mathrm{dl}$ and a reconstitution of the blood-CSF barrier. Pleocytosis dropped further after 10 more days to $18 / \mu$ l. Cytological examination of all 3 spinal CSF samples showed lymphocytic pleocytosis but no lymphoblasts or other atypical cells. The CSF/serum AI decreased after 7 days of treatment to 34.1 for IgM and 12.7 for IgG, and after a further 10 days to 17.4 for IgM and 15.6 for IgG. AIs were negative for Borrelia-specific IgG and further decreased for IgM (IgG negative in serum and CSF, IgM AI 6.5) after 4 months. The levels of CXCL13 were only slightly elevated in CSF and did not rise in the course of the current episode $(35 \mathrm{pg} / \mathrm{ml}$ at onset, 4.2 $\mathrm{pg} / \mathrm{ml}$ after 7 days, $4.5 \mathrm{pg} / \mathrm{ml}$ after another 10 days and $4.5 \mathrm{pg} / \mathrm{ml}$ after 4 months), which argues against acute neuroborreliosis during this episode. Pathological MEP became normal again after 20 days of treatment.

\section{Discussion}

The differential diagnostic for the relapsing spinal syndromes of our patient comprises spinal lymphoma, a spinal manifestation of X-type histiocytosis, neuromyelitis optica, multiple sclerosis (MS) or a systemic autoimmune disease. As our patient presented with only spinal and brainstem symptoms and no supratentorial lesions were detectable in MRI, the diagnostic criteria for MS were not fulfilled [7]. The 
long asymptomatic course between relapses, the absence of atypical cells in CSF, the positive response to antibiotic treatment (even without corticosteroids in 2008) and the serological findings argue against lymphoma. A relapse of X-type histiocytosis after 14 years without symptoms seems very improbable as the microscopic analysis of CSF revealed no evidence of histiocytes, and myelitis as a manifestation of histiocytosis is an extremely rare event, even though it has been previously reported [8]. The severe pleocytosis and damage to the BBB argue against neuromyelitis optica, MS or vasculitis especially since the visual system was unaffected and aquaporin-4 antibodies, oligoclonal bands, MRZ reaction, supratentorial MRI and the laboratory screening for vasculitis were negative.

The fact that a positive B. burgdorferi CSF/serum AI was found in 3 out of 4 episodes and that CSF pleocytosis and clinical symptoms ameliorated in response to antibiotic treatment seems to suggest recurrent episodes of neuroborreliosis. However, recurrent episodes of borreliosis and even more so neuroborreliosis after sufficient antibiotic treatment such as in this case, are very uncommon. If relapses occur, they are attributed to reinfections or relapses after inadequate antibiotic therapy. Moreover, it needs to be considered that the patient did not recall any previous tick bite or erythema migrans nor does she belong to a part of the population with an increased risk for repeated tick bites. Further, it remains unclear why the AI was negative during the third episode while all other signs and symptoms were similar. All 4 episodes manifested with a spinal syndrome despite the fact that $B$. burgdorferi myelitis represents less than $5 \%$ of cases with neuroborreliosis in larger studies [9-11]. Positive CSF/serum IgG AI can be found after a successfully treated and no longer active neuroborreliosis. But we would expect continuously positive predominantly IgG AIs in the case of AI persistence after an acute infection or during a persisting or remitting infection involving the CNS. However, this was not the case during the episode in 2008 when the AI was normal or in the current episode when AIs returned to normal 3 months after the start of the episode.

AIs can change over time depending on the activation status of the immune system. Increasing AIs are not specific for an acute borreliosis as they can also be the result of an unspecific stimulation of the immune system, e.g. by an autoimmune manifestation or a yet unidentified infection in our patient. AIs are calculated as the ratio of specific to total immunoglobulins in CSF divided by the ratio of specific to total immunoglobulins in serum. It is therefore possible that low concentrations of total immunoglobulins in CSF give rise to positive AIs at rather low specific concentrations in CSF, like the Borrelia-specific IgG concentration of $1.3 \mathrm{U} / \mathrm{ml}$ observed in our patient. In line with this, a Reiber diagram gave no evidence of intrathecal IgG synthesis in our patient.

Therefore, further parameters were needed to resolve the diagnostic dilemma in this case. Real-time PCR-based results for B. burgdorferi in CSF are often negative, as are cultures, even in the case of active neuroborreliosis; however, positive results can serve to ascertain the diagnosis. Thus, negative PCR results do not exclude neuroborreliosis in this case.

The level of CXCL13 in CSF is a very sensitive parameter for neuroborreliosis and can be of great help, especially in complex cases like ours $[12,13]$. In several studies using the same ready-made ELISA from R\&D Systems as in our patient, untreated neuroborreliosis could be differentiated from other neurological disorders with a high 
sensitivity of above $90 \%$ using cutoff values of CXCL13 concentrations in CSF of 250 $\mathrm{pg} / \mathrm{ml}$ [14], $100 \mathrm{pg} / \mathrm{ml}$ [15] or $142 \mathrm{pg} / \mathrm{ml}$ [16]. In a prospective trial, where a self-made ELISA with different binding properties was used, a sensitivity of $94.1 \%$ and a specificity of $96.1 \%$ were obtained with a cutoff of $1,229 \mathrm{pg} / \mathrm{ml}$, and sensitivity even reached $100 \%$ at a cutoff of $697 \mathrm{pg} / \mathrm{ml}$ [12]. An important finding of this large trial was that the CXCL13 concentration in CSF could discriminate neuroborreliosis from MS. The CXCL13 levels found in MS were only moderately elevated, like in our case. A reanalysis of the data of this prospective study revealed a negative predictive value of $99 \%$ for acute untreated neuroborreliosis (A. Plate, pers. commun.).

This large body of evidence clearly indicates a good negative predictive value for the CXCL13 concentration of $35 \mathrm{pg} / \mathrm{ml}$ observed in our patient. Thus, the recurrence of episodes despite sufficient antibiotic therapy and the fact that she did not recall a tick bite or erythema migrans and did not belong to a risk group for repeated tick bites strongly argue against acute neuroborreliosis as the cause of our patient's current episode.

Instead, it seems most probable that our patient had suffered from neuroborreliosis in the past, e.g. when she had her first episodes of neurological symptoms in 1996 and/or 1999, which was effectively treated and cured back then. We interpret the subsequent episodes in 2008 and 2011 as post-infectious autoimmune-mediated transverse myelitis triggered by the preceding neuroborreliosis. Immune responses specific against CNS proteins or myelin have been reported in CSF of neuroborreliosis patients [17-22], and post-infectious autoimmune mechanisms following Lyme borreliosis have been discussed for arthritis and autoimmune polyneuropathy [23, 24]. So an autoimmune mechanism caused by molecular mimicry seems a plausible explanation for the relapsing events of myelitis observed in our patient. The fact that she recovered in 2008 even without steroid treatment does not argue against an autoimmune transverse myelitis, as these disorders are also known to show spontaneous remissions, albeit with a slower recovery than with steroid treatment. Also, the fact that we did not observe more bands in the B. burgdorferi Western blot in CSF and serum argues against a persistent and relapsing neuroborreliosis, in which we would expect more bands, particularly IgG. The findings are in line with our assumption that our patient had contact with B. burgdorferi before, possibly even neuroborreliosis during one of the first episodes. Thus, the bands detectable in CSF and serum during the fourth episode would have been caused by a persistence of specific B cells, which are stimulated in the context of the presumed autoimmune event.

Taken together, our case highlights the diagnostic challenges that may occur in patients with inflammatory lesions of the spinal cord and/or positive B. burgdorferi serology.

\section{Disclosure Statement}

The authors declare no conflicts of interests or financial disclosures. 

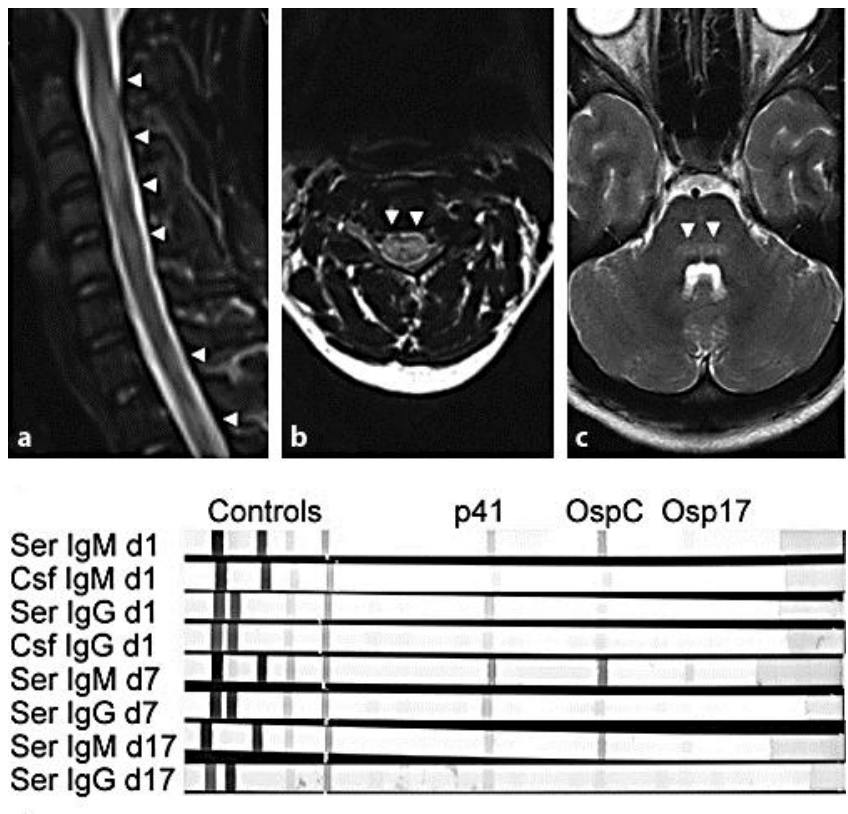

d

Fig. 1. Extensive high-signal alterations are visible in the cervical and thoracic spinal cord, comprising large portions of the medullar cross section (sagittal STIR, short-tau inversion recovery sequence, a; axial $\mathrm{T}_{2}$-weighted turbo spin echo sequence, b). c Cerebral involvement is also seen as bilateral hyperintense signal alterations in the dorsal portions of the pons (axial $\mathrm{T}_{2}$-weighted turbo spin echo sequence). $\mathbf{d}$ Western blots for IgG and IgM of serum and CSF at day 1 (d1) and serum at day 7 (d7) and day 17 (d17). Bands before the blue alignment marker are positive controls for serum, IgG, IgM and a cutoff control (in this order). The Borrelia-specific antigens p41, OspC, and Osp17 are indicated. The VlsE antigen showed no band in any of the samples.

\section{References}

1 Fahrer H, Sauvain MJ, vd Linden S, Zhioua E, Gern L, Aeschlimann A: Prevalence of Lyme borreliosis in a Swiss population at risk (in German). Schweiz Med Wochenschr 1988;118:65-69.

$\checkmark 2$ Kaiser R, Kern A, Kampa D, Neumann-Haefelin D: Prevalence of antibodies to Borrelia burgdorferi and tick-borne encephalitis virus in an endemic region in southern Germany. Zentralbl Bakteriol 1997;286:534-541.

-3 Stanek G, Flamm H, Groh V, Hirschl A, Kristoferitsch W, Neumann R, et al: Epidemiology of borrelia infections in Austria. Zentralbl Bakteriol Mikrobiol Hyg A 1987;263:442-449.

-4 Stanek G, Wormser GP, Gray J, Strle F: Lyme borreliosis. Lancet 2012;379:461-473.

5 Pfister HW, Wilske B, Weber K: Lyme borreliosis: basic science and clinical aspects. Lancet 1994;343:1013-1016.

6 Steere AC, Coburn J, Glickstein L: The emergence of Lyme disease. J Clin Invest 2004;113:1093-1101.

-7 Polman CH, Reingold SC, Banwell B, Clanet M, Cohen JA, Filippi M, et al: Diagnostic criteria for multiple sclerosis: 2010 revisions to the McDonald criteria. Ann Neurol 2010;69:292-302.

-8 Cardozo LJ, Bailey IC, Billinghurst JR, Poltera AA: Non-osseous eosinophilic granuloma presenting as acute transverse myelitis. Br J Surg 1974;61:747-749.

$\checkmark 9$ Hansen K, Lebech AM: The clinical and epidemiological profile of Lyme neuroborreliosis in Denmark 1985-1990. A prospective study of 187 patients with Borrelia burgdorferi-specific intrathecal antibody production. Brain 1992;115(Pt 2):399-423.

10 Oschmann P, Dorndorf W, Hornig C, Schafer C, Wellensiek HJ, Pflughaupt KW: Stages and syndromes of neuroborreliosis. J Neurol 1998;245:262-272. 
11 Huppertz HI, Bohme M, Standaert SM, Karch H, Plotkin SA: Incidence of Lyme borreliosis in the Würzburg region of Germany. Eur J Clin Microbiol Infect Dis 1999;18:697-703.

12 Schmidt C, Plate A, Angele B, Pfister HW, Wick M, Koedel U, et al: A prospective study on the role of CXCL13 in Lyme neuroborreliosis. Neurology 2011;76:1051-1058.

-13 Kingwell K: Infectious disease: CXCL13 is a potential biomarker for Lyme neuroborreliosis. Nature Reviews 2011;7:244.

14 van Burgel ND, Bakels F, Kroes AC, van Dam AP: Discriminating Lyme neuroborreliosis from other neuroinflammatory diseases by levels of CXCL13 in cerebrospinal fluid. J Clin Microbiol 2011;49:20272030

15 Wutte N, Berghold A, Loffler S, Zenz W, Daghofer E, Krainberger I, Kleintert G, Aberer E: CXCL13 chemokine in pediatric and adult neuroborreliosis. Acta Neurol Scand 2011;124:321-328.

16 Senel M, Rupprecht TA, Tumani H, Pfister HW, Ludolph AC, Brettschneider J: The chemokine CXCL13 in acute neuroborreliosis. J Neurol Neurosurg Psychiatry 2010;81:929-933.

$\checkmark 17$ Kuenzle S, von Budingen HC, Meier M, Harrer MD, Urich E, Becher B, Goebels N: Pathogen specificity and autoimmunity are distinct features of antigen-driven immune responses in neuroborreliosis. Infect Immun 2007;75:3842-3847.

18 Kaiser R: Intrathecal immune response in patients with neuroborreliosis: specificity of antibodies for neuronal proteins. J Neurol 1995;242:319-325

19 Baig S, Olsson T, Hojeberg B, Link H: Cells secreting antibodies to myelin basic protein in cerebrospinal fluid of patients with Lyme neuroborreliosis. Neurology 1991;41:581-587.

-20 Schluesener HJ, Martin R, Sticht-Groh V: Autoimmunity in Lyme disease: molecular cloning of antigens recognized by antibodies in the cerebrospinal fluid. Autoimmunity 1989;2:323-330.

-21 Pohl-Koppe A, Logigian EL, Steere AC, Hafler DA: Cross-reactivity of Borrelia burgdorferi and myelin basic protein-specific $\mathrm{T}$ cells is not observed in borrelial encephalomyelitis. Cell Immunol 1999;194:118-123.

-22 Martin R, Ortlauf J, Sticht-Groh V, Mertens HG: Isolation and characterization of Borrelia burgdorferispecific and autoreactive T-cell lines from the cerebrospinal fluid of patients with Lyme meningoradiculomyelitis. Ann NY Acad Sci 1988;540:449-451.

23 Drouin EE, Glickstein L, Kwok WW, Nepom GT, Steere AC: Searching for borrelial T cell epitopes associated with antibiotic-refractory Lyme arthritis. Mol Immunol 2008;45:2323-2332.

-24 Rupprecht TA, Elstner M, Weil S, Pfister HW: Autoimmune-mediated polyneuropathy triggered by borrelial infection? Muscle Nerve 2008;37:781-785. 